\title{
Measurement of Esophageal Nocturnal Baseline Impedance: A Simplified Method
}

\author{
Yoshimasa Hoshikawa, Akinari Sawada, Shirley Sonmez, Kornilia Nikaki, Philip Woodland, Etsuro Yazaki, and Daniel Sifrim* \\ Barts and the London School of Medicine and Dentistry, Queen Mary University of London, UK
}

\section{Background/Aims}

Mean nocturnal baseline impedance (MNBI) during multichannel intraluminal impedance pH-monitoring (MII-pH) reflects the status of esophageal mucosal integrity. MNBI is suggested as an adjunctive method to distinguish patients with true gastroesophageal reflux disease (GERD) from functional heartburn (FH) and might predict outcomes for anti-reflux treatment. However, current methodology for calculation of MNBI is time-consuming and subject to operator-dependent selection bias. We aim to simplify and provide a more objective method to calculate MNBI.

\section{Methods}

We retrospectively analyzed $100 \mathrm{MII}-\mathrm{pH}$ tracings from 20 patients with erosive reflux disease, 20 with non-erosive reflux disease (NERD), 20 with reflux hypersensitivity, 20 with functional heartburn (FH), and 20 healthy asymptomatic volunteers. We compared the current "conventional" MNBI analysis with our "simple" MNBI analysis measured by selecting the whole supine period using the impedance average calculation function in the MII-pH software.

\section{Results}

Absolute values were very similar and there was a strong correlation between conventional and simple MNBI values in the most distal channel in all groups $(r \geq 0.8, P<0.001)$ including patients with increased supine acid reflux. Distal esophageal simple MNBI negatively correlated with acid exposure time $(r=-0.695, P<0.001)$. Patients with erosive reflux disease and NERD had lower simple MNBI values in the most distal channel compared to other groups $(P<0.001)$. With a cutoff value of $1785 \mathrm{ohms}$, simple MNBI can discriminate patients with GERD from those with reflux hypersensitivity and FH (sensitivity $80.0 \%$ and specificity $89.7 \%$ ).

\section{Conclusion}

Simple MNBI analysis provides very similar values and has an excellent correlation with conventional MNBI analysis.

(J Neurogastroenterol Motil 2020;26:241-247)

Key Words

Electric impedance; Esophageal mucosa; Esophagitis; Gastroesophageal reflux; Heartburn

\section{Introduction}

Esophageal multichannel intraluminal impedance $\mathrm{pH}-$ monitoring (MII-pH) is often performed in patients with reflux symptoms to confirm presence of gastroesophageal reflux disease (GERD) when the symptoms are refractory to proton pump inhibitors (PPI) or when endoscopic/surgical interventions are consid-

Received: September 19, 2019 Revised: December 6, 2019 Accepted: December 12, 2019

(a) This is an Open Access article distributed under the terms of the Creative Commons Attribution Non-Commercial License (http://creativecommons. org/licenses/by-nc/4.0) which permits unrestricted non-commercial use, distribution, and reproduction in any medium, provided the original work is properly cited.

${ }^{*}$ Correspondence: Daniel Sifrim, MD PhD

Wingate Institute of Neurogastroenterology, 26 Ashfield Street, Whitechapel, London E1 2AJ, UK

Tel: +44-0-20-7882-5555, E-mail: d.sifrim@qmul.ac.uk 
ered. ${ }^{1,2}$ Although total acid exposure time (AET) is used as a predictive factor for anti-reflux therapies, it has significant day-to-day variability. $^{1,3-5}$ In contrast, other parameters from reflux monitoring might be more stable over time and reflect the status of esophageal mucosal integrity.

Measurement of esophageal baseline impedance (BI) reflects esophageal mucosal integrity and correlates well with levels of AET and acid sensitivity. ${ }^{6-8}$ Baseline esophageal impedance measurements continuously changes over time due to occurrence of swallows and reflux of liquids and gas. During sleep periods, such changes are minimized. Mean nocturnal baseline impedance (MNBI) was suggested as an accurate method to characterize BI. ${ }^{9,10}$ MNBI can distinguish different GERD phenotypes from reflux-unrelated symptoms (functional heartburn $[\mathrm{FH}]$ ) and provides a good predictive value for anti-reflux therapies. ${ }^{9-16}$ However, calculation of MNBI is time-consuming, particularly in patients with supine frequent swallowing and reflux. Furthermore, automatic calculation of MNBI is currently not included in most commercially available MII-pH software. We aim to simplify the method to measure MNBI.

\section{Materials and Methods}

\section{Subjects}

This was a single-center retrospective case-control study. We analyzed a total of 100 MII-pH tracings obtained from our database at Upper GI Physiology Unit, 20 patients with erosive reflux disease (ERD) (6 grade A, 12 grade $B$, and 2 grade $C$ ), 20 patients with non-erosive reflux disease (NERD), 20 patients with reflux hypersensitivity (RH), 20 patients with $\mathrm{FH}$ and 20 healthy asymptomatic volunteers (HV). Eligibility criteria for patients were (1) presence of typical reflux symptoms which were refractory to at least 8-week double dose PPI treatment, (2) an endoscopy performed within 2 years prior to MII-pH, and (3) each group was defined based on Lyon Consensus and Rome IV criteria: ERD diagnosed by an endoscopy, NERD defined as a negative endoscopy and pathological AET ( $>6 \%$ ), RH defined as a negative endoscopy, physiological AET $(<4 \%)$, and positive symptom index or symptom association probability, $\mathrm{FH}$ defined as a negative endoscopy, physiological AET $(<4 \%)$, and negative symptom association probability/symptom index. ${ }^{1,17}$ Patients with Barrett's esophagus were excluded. HV did not have relevant medical history, upper abdominal surgery, or pathological reflux at MII-pH investigation. MII-pH measurements were performed between 2017 and 2019 and were re-analyzed as part of this study. Our study adhered to the principles outlined in the Declaration of Helsinki. As this is a retrospective analysis with no identifiable patient data and all tests were performed as clinically indicated, formal ethics approval was not deemed as necessary, but we obtained approval from our Quality and Service Improvement department at Royal London Hospital, Barts Health NHS trust.

\section{Multichannel intraluminal impedance pH-monitoring protocol}

Patients were instructed to stop PPI and histamine $\mathrm{H} 2$ blocker for at least 7 days prior to the study. After overnight fast, patients underwent High-resolution manometry (Medtronic, Minneapolis MN, USA) to detect the proximal margin of the lower esophageal sphincter (LES) and check the existence and length of a hiatus hernia. ${ }^{18}$ Then, we placed the MII-pH catheter (Diversatek Healthcare, Highlands Ranch CO, USA or OMOM, Jinshan Science and Technology, Chongqing, China) with a distal $\mathrm{pH}$ sensor at $5 \mathrm{~cm}$ above the LES. The catheter has 6 impedance pairs of electrodes at $3,5,7,9,15$, and $17 \mathrm{~cm}$ above the LES and $2 \mathrm{pH}$ sensors. We analyzed the MII-pH tracings using the dedicated softwares (Diversatek Healthcare or Jinshan Science and Technology) and visual editing based on our standard protocol. ${ }^{1,19,20}$

\section{Mean nocturnal baseline impedance}

We calculated MNBI values in the distal esophagus (at $3 \mathrm{~cm}$ and $5 \mathrm{~cm}$ above the LES) using 2 methods: "conventional" MNBI reported by Martinucci et al, ${ }^{9}$ and a simplified method (simple MNBI). The Chonqing method involved manual measurement of mean $\mathrm{BI}$ values at 3 different stable 10-minute time periods (around $1 \mathrm{AM}, 2 \mathrm{AM}$, and $3 \mathrm{AM}$ ), excluding reflux episodes and swallowing. Then, the values were averaged to obtain Chonqing MNBI. Simple MNBI was measured during the whole supine period using the automatic calculation function in the software (Fig. 1). If there were 2 or more recumbent period markers, we chose the longest one. These analyses were performed blindly to subjects' conditions.

\section{Data analysis and statistical analysis}

All continuous variables are presented as median with interquartile range and assumed not to have a normal distribution. Fisher exact test was used to compare the ratios. Continuous variables were compared using Mann-Whitney $U$ test or Kruskal-Wallis test followed by Bonferroni correction for multiple comparisons. Correlation between continuous variables was tested using Spearman's rank correlation test. Diagnostic accuracy of both MNBI values at $5 \mathrm{~cm}$ and $3 \mathrm{~cm}$ above the LES were assessed using a receiver 


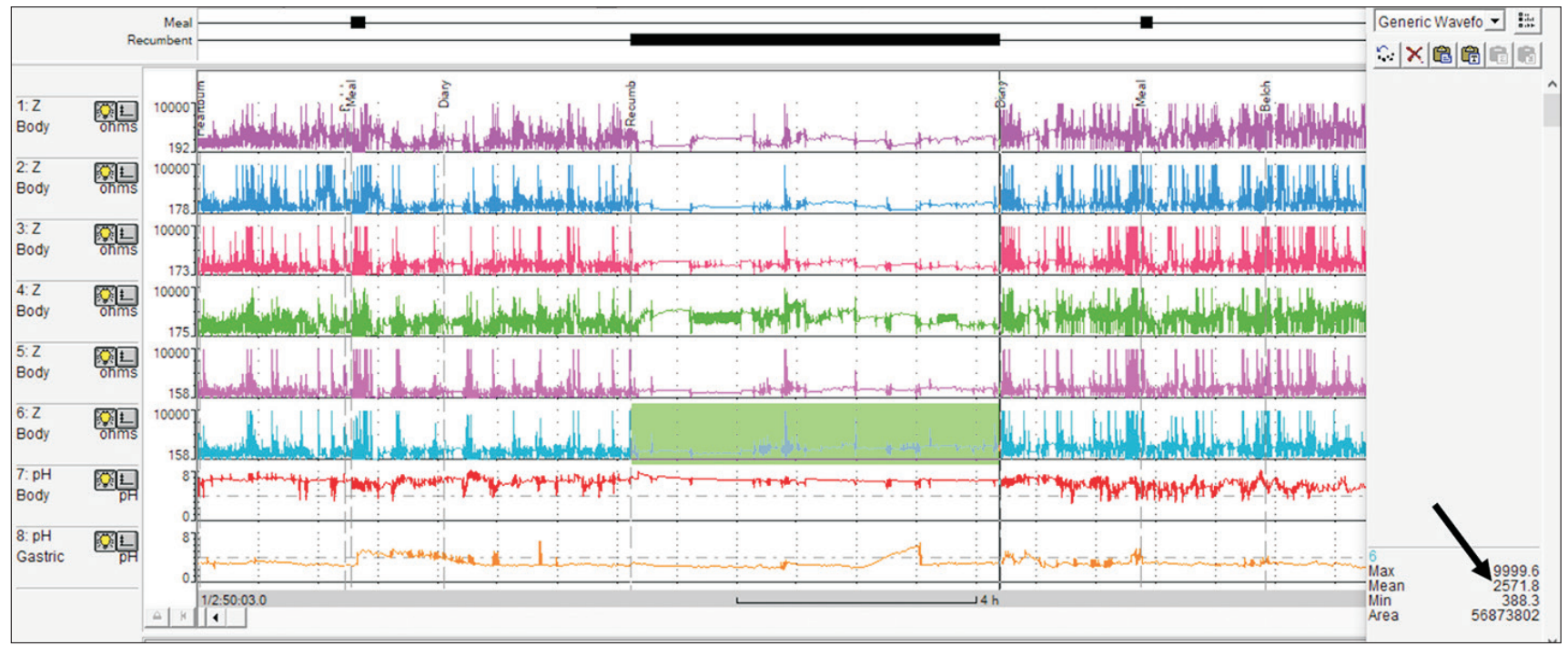

Figure 1. Simple mean nocturnal baseline impedance (MNBI). New method to measure MNBI by selecting whole recumbent period and using the automatic impedance average calculation function provided by commercial software to measure MNBI. The arrow indicates the MNBI value in the most distal channel in this patient.

Table 1. Characteristics of All Groups

\begin{tabular}{|c|c|c|c|c|c|c|}
\hline Characteristics & $\operatorname{ERD}(\mathrm{n}=20)$ & $\operatorname{NERD}(\mathrm{n}=20)$ & $\mathrm{RH}(\mathrm{n}=20)$ & $\mathrm{FH}(\mathrm{n}=20)$ & $\mathrm{HV}(\mathrm{n}=20)$ & $P$-value \\
\hline Age (yr) & $56.5(51.0-64.3)$ & $53.0(46.8-62.3)$ & $39.0(35.8-49.8)$ & $43.0(34.0-51.3)$ & $37.5(31.0-42.8)$ & $<0.001$ \\
\hline Gender (female) & $30 \%$ & $55 \%$ & $60 \%$ & $60 \%$ & $60 \%$ & 0.263 \\
\hline BMI & $27.0(24.9-29.7)$ & $26.7(23.3-28.8)$ & $28.5(23.0-30.1)$ & $24.6(22.5-30.0)$ & ND & 0.933 \\
\hline Hiatus hernia & $80 \%$ & $30 \%$ & $30 \%$ & $25 \%$ & ND & $<0.001$ \\
\hline $\begin{array}{l}\text { Total acid } \\
\text { exposure time }(\%)\end{array}$ & $9.6(7.9-17.2)$ & $8.9(8.2-11.9)$ & $1.9(1.1-2.7)$ & $0.7(0.2-1.6)$ & $0.7(0.1-1.2)$ & $<0.001$ \\
\hline $\begin{array}{l}\text { Upright acid } \\
\text { exposure time (\%) }\end{array}$ & $13.2(9.6-16.5)$ & $10.7(5.8-14.6)$ & $3.2(1.5-4.8)$ & $1.0(0.3-2.1)$ & $0.6(0.2-1.8)$ & $<0.001$ \\
\hline $\begin{array}{l}\text { Recumbent acid } \\
\text { exposure time (\%) }\end{array}$ & $4.1(0.2-15.9)$ & $7.7(2.5-13.8)$ & $0.2(0.0-0.9)$ & $0.2(0.0-0.5)$ & $0.0(0.0-0.6)$ & $<0.001$ \\
\hline Total reflux episodes & $61.5(42.8-101.3)$ & $44.0(32.3-70.0)$ & $47.5(34.0-55.8)$ & $18.5(9.8-29.3)$ & $33.0(20.3-44.8)$ & $<0.001$ \\
\hline $\begin{array}{l}\text { Conventional MNBI } \\
\text { at } 17 \mathrm{~cm}\end{array}$ & $2540(2331-2961)$ & $2639(1814-3716)$ & $2732(2389-3263)$ & $2992(2620-3902)$ & $2169(1325-3036)$ & 0.186 \\
\hline $\begin{array}{l}\text { Conventional MNBI } \\
\text { at } 15 \mathrm{~cm}\end{array}$ & $2030(1688-2537)$ & $2415(1445-2810)$ & $2267(1983-2783)$ & $2680(2400-3121)$ & $1866(1027-2434)$ & 0.037 \\
\hline $\begin{array}{l}\text { Conventional MNBI } \\
\text { at } 9 \mathrm{~cm}\end{array}$ & $1988(1481-2383)$ & $1465(1236-2257)$ & $2222(1904-2849)$ & $2673(2193-3196)$ & $2656(2349-3038)$ & 0.003 \\
\hline $\begin{array}{l}\text { Conventional MNBI } \\
\text { at } 7 \mathrm{~cm}\end{array}$ & $1419(1179-2185)$ & $1460(1036-2396)$ & $2708(2297-3159)$ & $3247(2467-4381)$ & $3094(2522-4360)$ & $<0.001$ \\
\hline $\begin{array}{l}\text { Conventional MNBI } \\
\text { at } 5 \mathrm{~cm}\end{array}$ & $969(750-1652)$ & $1229(816-2058)$ & $2867(2392-3440)$ & 3035 (1692-3152) & $2765(1692-3152)$ & $<0.001$ \\
\hline $\begin{array}{l}\text { Conventional MNBI } \\
\text { at } 3 \mathrm{~cm}\end{array}$ & $854(509-1318)$ & $1370(812-1773)$ & $2631(1970-3680)$ & $3200(2270-4113)$ & $2662(1971-3344)$ & $<0.001$ \\
\hline
\end{tabular}

ERD, erosive reflux disease; NERD, non-erosive reflux disease; RH, reflux hypersensitivity; FH, functional heartburn; HV, healthy volunteer; BMI, body mass index; MNBI, mean nocturnal baseline impedance; ND, no data.

Value are given as median (interquartile range). 
operating characteristics (ROC) analysis. DeLong's test was performed to compare the 2 ROC curves. All statistical analyses were performed with EZR (version 1.36; Saitama Medical Center Jichi Medical University, Saitama, Japan), which is a graphical user interface for R (The R Foundation for Statistical Computing, Vienna, Austria). ${ }^{21}$

\section{Results}

Patients with ERD and NERD were older than patients with $\mathrm{RH}, \mathrm{FH}$, and $\mathrm{HV}$ and by definition had significantly higher esophageal acid exposure. Patients with ERD had higher prevalence of hiatus hernia (Table 1). Using conventional MNBI measurements, patients with ERD and NERD showed lower MNBI values than patients with $\mathrm{RH}, \mathrm{FH}$, and $\mathrm{HV}$ at $3 \mathrm{~cm}$ and $5 \mathrm{~cm}$ above the LES.

\section{Conventional vs Simple Mean Nocturnal Baseline Impedance}

Absolute values of MNBI in the distal esophagus were very similar when calculated using either conventional or simple measurement methods. The median percent difference between absolute values was $11-12 \%$. There was a strong correlation between conventional MNBI and simple MNBI values in all channels among all subjects ( $\mathrm{n}=100, r \geq 0.8, P<0.001$ in all channels) (Table 2). More importantly, there was a strong correlation between conventional MNBI and simple MNBI values in the most distal impedance channel and this was true for all groups including patients with esophagitis (Table 3). When subjects were divided into those with pathological supine acid exposure $(\text { AET }>2.1 \%)^{20}(\mathrm{n}=30)$ and subjects with normal supine acid exposure $(\mathrm{n}=70)$, we found a very good correlation between conventional and simple MNBI values $(r=0.96$ and 0.91 respectively, $P<0.001$ for both).

Both, conventional and simple MNBI values in the distal impedance channel negatively correlated with AET $(r=-0.648$ and
-0.695 respectively, $P<0.001$ for both)

\section{Comparison of Simple Mean Nocturnal Baseline Impedance Values in the Distal Esophagus Among Groups}

Table 4 shows simple MNBI median values in all groups. simple MNBI values in the most distal channel showed the same trends as conventional MNBI. Patients with ERD and NERD had significantly lower baseline impedance values than patients with $\mathrm{RH}, \mathrm{FH}$, and HV (Fig. 2).

\section{Diagnostic Accuracy of Simple and Conventional Mean Nocturnal Baseline Impedance to Discriminate Patients With Erosive Reflux Disease and "True" Non-erosive Reflux Disease}

We assessed accuracy of both methods (measuring MNBI at $5 \mathrm{~cm}$ and $3 \mathrm{~cm}$ above LES) for diagnosis of GERD (ERD and NERD) using ROC analysis. The area under curves were 0.881 (95\% CI, 0.802-0.960) and 0.895 (95\% CI, 0.824-0.966) with simple MNBI at $5 \mathrm{~cm}$ and $3 \mathrm{~cm}$ above the LES respectively, and 0.854 (95\% CI, 0.765-0.949) and 0.872 (95\% CI, 0.790-0.955) with conventional MNBI. Using a cutoff value of $1785 \mathrm{ohms}$ at 3 $\mathrm{cm}$ above the LES, conventional method had a sensitivity of $82.5 \%$

Table 3. Correlation Between Conventional and Simple Mean Nocturnal Baseline Impedance Values in the Most Distal Channel in Each Group

\begin{tabular}{lrr}
\hline \multicolumn{1}{c}{ Group } & $r$-value & $P$-value \\
\hline ERD & 0.973 & $<0.001$ \\
NERD & 0.967 & $<0.001$ \\
RH & 0.842 & $<0.001$ \\
FH & 0.862 & $<0.001$ \\
HV & 0.886 & $<0.001$ \\
\hline
\end{tabular}

ERD, erosive reflux disease; NERD, non-erosive reflux disease; $\mathrm{RH}$, reflux hypersensitivity; $\mathrm{FH}$, functional heartburn; $\mathrm{HV}$, healthy volunteer.

Table 2. Correlation Between Conventional and Simple Mean Nocturnal Baseline Impedance Values in All Channels $(\mathrm{N}=100)$

\begin{tabular}{|c|c|c|c|c|c|}
\hline \multirow{2}{*}{ Channel } & \multicolumn{2}{|c|}{ Correlation } & \multicolumn{3}{|c|}{ Absolute values (median) } \\
\hline & Correlation coefficient & $P$-value & Simple MNBI & Conventional MNBI & $P$-value \\
\hline At $17 \mathrm{~cm}$ & 0.85 & $<0.001$ & 2698 & 2671 & 0.668 \\
\hline At $15 \mathrm{~cm}$ & 0.80 & $<0.001$ & 2295 & 2332 & 0.985 \\
\hline At $9 \mathrm{~cm}$ & 0.86 & $<0.001$ & 2155 & 2270 & 0.755 \\
\hline At $7 \mathrm{~cm}$ & 0.90 & $<0.001$ & 2311 & 2461 & 0.526 \\
\hline At $5 \mathrm{~cm}$ & 0.95 & $<0.001$ & 2304 & 2350 & 0.702 \\
\hline At $3 \mathrm{~cm}$ & 0.95 & $<0.001$ & 2258 & 2193 & 0.829 \\
\hline
\end{tabular}

MNBI, mean nocturnal baseline impedance. 
Table 4. Simple Mean Nocturnal Baseline Impedance Values Among All Groups

\begin{tabular}{lcllll}
\hline \multicolumn{1}{c}{ Channel } & ERD $(\mathrm{n}=20)$ & NERD $(\mathrm{n}=20)$ & RH $(\mathrm{n}=20)$ & FH $(\mathrm{n}=20)$ & HV $(\mathrm{n}=20)$ \\
\hline Simple MNBI at $17 \mathrm{~cm}$ & $2529(2281-2947)$ & $2830(2016-3424)$ & $2830(2283-3159)$ & $2987(2129-3736)$ & $2129(2016-3424)$ \\
Simple MNBI at $15 \mathrm{~cm}$ & $2198(1968-2492)$ & $2652(1890-2882)$ & $2440(2174-2778)$ & $2641(1915-3344)$ & $2002(1193-2121)$ \\
Simple MNBI at $9 \mathrm{~cm}$ & $2010(1441-2010)$ & $1438(1198-2027)$ & $2307(2007-2721)$ & $2582(2294-3710)$ & $2439(2152-2984)$ \\
Simple MNBI at $7 \mathrm{~cm}$ & $1446(1122-1964)$ & $1506(1043-2174)$ & $2428(2143-3249)$ & $3019(2487-4048)$ & $2960(2454-4055)$ \\
Simple MNBI at $5 \mathrm{~cm}$ & $992(788-1598)$ & $1192(854-2153)$ & $2543(2150-3282)$ & $3127(2491-3904)$ & $2751(2269-2943)$ \\
Simple MNBI at $3 \mathrm{~cm}$ & $914(580-1273)$ & $1276(801-1797)$ & $2353(2009-3565)$ & $2932(2483-4175)$ & $2479(2055-3167)$ \\
\hline
\end{tabular}

ERD, erosive reflux disease; NERD, non-erosive reflux disease; RH, reflux hypersensitivity; FH, functional heartburn; HV, healthy volunteer; MNBI, mean nocturnal baseline impedance.

Value are given as median (interquartile range).

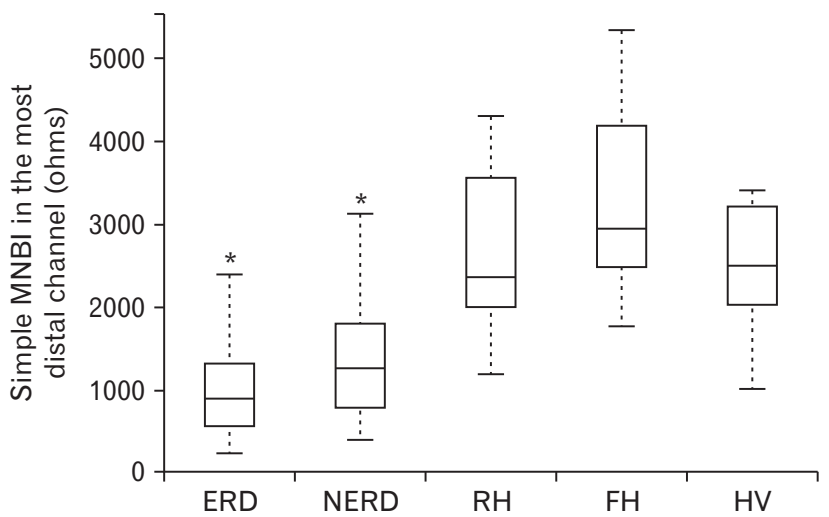

Figure 2. Simple mean nocturnal baseline impedance (MNBI) values in the most distal channel. Patients with erosive reflux disease (ERD) and non-erosive reflux disease (NERD) had lower values than other groups ( $\left.{ }^{*}<0.001\right)$. RH, reflux hypersensitivity; $\mathrm{FH}$, functional heartburn; HV, healthy volunteer.

and specificity of $89.7 \%$. When measured at $5 \mathrm{~cm}$ above the LES, conventional method with a cutoff value of $1943 \mathrm{ohms}$ had a sensitivity of $80.0 \%$ and a specificity of $90.0 \%$. Likewise, using a cutoff value of $1785 \mathrm{ohms}$ at $3 \mathrm{~cm}$ above the LES, simple MNBI analysis showed a sensitivity of $80.0 \%$ and specificity of $89.7 \%$. When calculated at $5 \mathrm{~cm}$, a cutoff value of $2034 \mathrm{ohms}$ had a sensitivity of $80.0 \%$ and specificity of $85.0 \%$ (Fig. 3).

Table 5 shows there were no significant differences in the proportions of patients who had lower MNBI values than the cutoff with both methods in GERD and non-GERD group.

\section{Discussion}

In this study, we have shown that (1) a simplified method to assess baseline impedance during 24 hours reflux monitoring, simple MNBI, correlated very well with the values obtained using the previously described method by Martinucci et al, " "conventional"
MNBI; a good correlation was found in all patient groups regardless of recumbent acid exposure time, (2) simple MNBI negatively correlated with AET in distal esophageal channels, (3) simple MNBI values were significantly lower in ERD and NERD as compared to patients with $\mathrm{FH}, \mathrm{RH}$, and $\mathrm{HV}$, and (4) a cutoff value of $1785 \mathrm{ohms}$ with simple MNBI at $3 \mathrm{~cm}$ above the LES can discriminate patients with GERD (ERD and NERD) with sensitivity of $80.0 \%$ and specificity of $89.7 \%$.

In our study, we defined the different groups of patients with PPI-refractory typical reflux symptoms using the recent, more stringent criteria suggested by the Lyon Consensus. ${ }^{1}$ The current consensus papers and guidelines for GERD management suggest an initial empirical PPI treatment followed by endoscopy and reflux monitoring if the patient does not respond to PPI. Therefore, the group of GERD patient most frequently seen in a tertiary referral center gastrointestinal physiology unit is a patient that was refractory to PPI empirical treatment.

Nevertheless, our simplified method to measure impedance baseline, simple MNBI, showed very similar values and correlations as compared to those from conventional analysis. ${ }^{7,14}$ Frazzoni et $\mathrm{al}^{22,23}$ reported that distal MNBI values in patients with $\mathrm{RH}$ is lower than those obtained in patients with $\mathrm{FH}$ and suggested that RH patients should be considered as part of the spectrum of reflux disease rather than a functional disorder. Our results did not support a significant difference in the MNBI between the 2 groups in both simple and conventional analysis.

The clinical usefulness of MNBI or BI is still controversial. Some authors suggest that it cannot yet be considered a full diagnostic tool of GERD whereas other authors reported that low BI can be a predictor of outcomes for anti-reflux therapies. ${ }^{10,15,16,24} \mathrm{In}$ the context of the new definition of GERD provided by the Lyon consensus, Rangarajan et al reported that low MNBI can identify those patients that will respond to anti-reflux therapies if they have a borderline acid exposure $(4 \% \leq \mathrm{AET} \leq 6 \%) .{ }^{24}$ In addition, it 
A

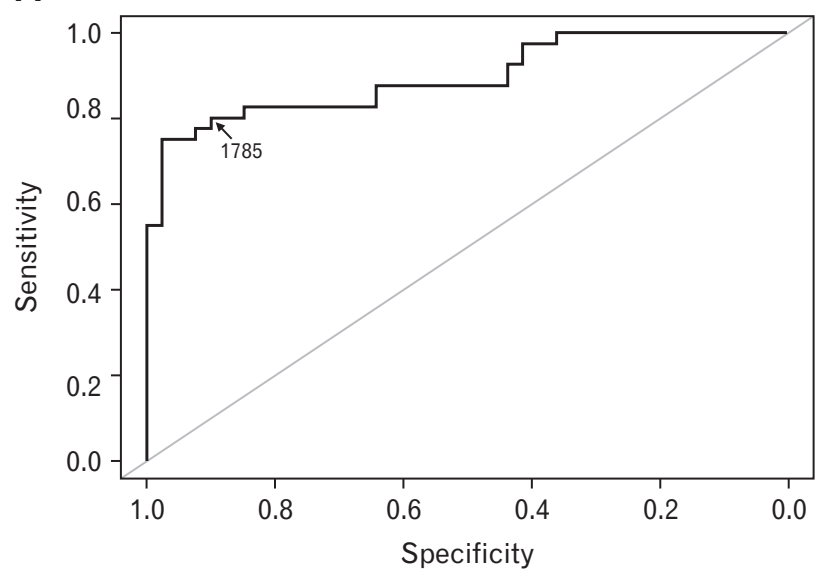

B

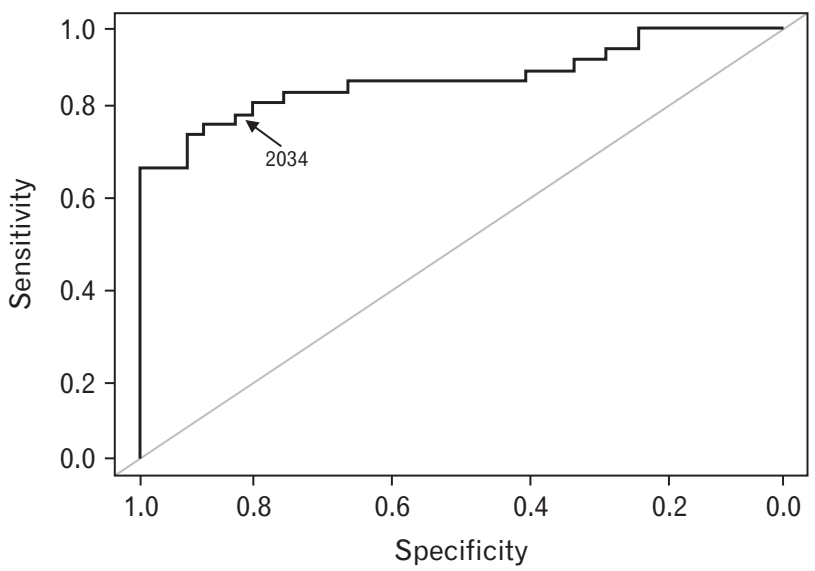

Figure 3. Receiver operating characteristics curves for simple mean nocturnal baseline impedance at $3 \mathrm{~cm}(\mathrm{~A})$ and $5 \mathrm{~cm}(\mathrm{~B})$ above the lower esophageal sphincter for diagnosis of gastroesophageal reflux disease. The area under curves were 0.895 (95\% CI, 0.824-0.966) and 0.881 (95\% CI, 0.802-0.960) respectively, $P=0.508$.

Table 5. Comparison of the Proportions of Patients Who Had Lower Mean Nocturnal Baseline Impedance Values Than the Cutoff With Both Methods in Gastroesophageal Reflux Disease and Non-gastroesophageal Reflux Disease Groups

\begin{tabular}{lcccccc}
\hline & \multicolumn{2}{c}{ MNBI value at $5 \mathrm{~cm}$ above the LES } & & \multicolumn{3}{c}{ MNBI value at $3 \mathrm{~cm}$ above the LES } \\
\cline { 2 - 5 } \cline { 5 - 7 } & Simple & Conventional & $P$-value & Simple & Conventional & $P$-value \\
\hline Cutoff value & 2034 & 1943 & & 1785 & 1784 \\
Non-GERD (FH + RH) & $6 / 40$ & $4 / 40$ & 0.737 & $4 / 40$ & $4 / 40$ \\
GERD (ERD + NERD) & $32 / 40$ & $32 / 40$ & 1 & $32 / 40$ & $33 / 40$ \\
\hline
\end{tabular}

MNBI, mean nocturnal baseline impedance; LES, lower esophageal sphincter; GERD, gastroesophageal reflux disease; FH, functional heart burn; RH, reflux hypersensitivity; ERD, erosive reflux disease; NERD, non-erosive reflux disease.

is possible that MNBI could be considered a longitudinal marker of reflux burden, whereas AET has important day-to-day variability. $^{1,4,8,15}$ We believe that our simplified method to measure MNBI will facilitate the use of this parameter in further clinical studies.

Our study has limitations. This was a single-center retrospective analysis with relatively small number of subjects yet strong statistical results. We did not compare inter-observer agreement between both analysis methods. It is already known that conventional MNBI analysis has a relatively high interobserver agreement value. ${ }^{10}$ In our Center, we use for clinical purpose 2 different MII$\mathrm{pH}$ systems and the clinical data obtained are comparable. We do not have studies using simultaneously the 2 different systems in 1 patient. In any case, the absolute values of MNBI and correlations between the conventional and simple MNBI measurements were similar between the different MII-pH systems.

In summary, our study shows that simple MNBI analysis has an excellent correlation with previously described conventional MNBI analysis. We believe that the new method takes no more than 20 seconds, can prevent a bias and inter-individual variability in selection of periods of analysis, and may prove to be very useful in both clinical and research analysis of MII-pH.

\section{Financial support: None.}

\section{Conflicts of interest: None.}

Author contributions: Yoshimasa Hoshikawa performed analysis of MII tracings, interpretation of the results, and drafting the manuscript; Akinari Sawada, Shirley Sonmez, Kornilia Nikaki, Philip Woodland, and Etsuro Yazaki performed and analyzed the physiology studies; and Daniel Sifrim was involved in design of the study, interpretation of results and drafting of the final manuscript.

\section{References}

1. Gyawali CP, Kahrilas PJ, Savarino E, et al. Modern diagnosis of GERD: the Lyon Consensus. Gut 2018;67:1351-1362. 
2. Vaezi MF, Sifrim D. Assessing old and new diagnostic tests for gastroesophageal reflux disease. Gastroenterology 2018;154:289-301.

3. Scarpulla G, Camilleri S, Galante P, Manganaro M, Fox M. The impact of prolonged $\mathrm{pH}$ measurements on the diagnosis of gastroesophageal reflux disease: 4-day wireless $\mathrm{pH}$ studies. Am J Gastroenterol 2007;102:2642-2647.

4. Savarino E, Tutuian R, Zentilin P, et al. Characteristics of reflux episodes and symptom association in patients with erosive esophagitis and nonerosive reflux disease: study using combined impedance-pH off therapy. Am J Gastroenterol 2010;105:1053-1061.

5. de Bortoli N, Martinucci I, Savarino E, et al. Proton pump inhibitor responders who are not confirmed as GERD patients with impedance and pH monitoring: who are they? Neurogastroenterol Motil 2014;26:28-35.

6. Farré R, Blondeau K, Clement D, et al. Evaluation of oesophageal mucosa integrity by the intraluminal impedance technique. Gut 2011;60:885892.

7. Kessing BF, Bredenoord AJ, Weijenborg PW, Hemmink GJ, Loots $\mathrm{CM}$, Smout AJ. Esophageal acid exposure decreases intraluminal baseline impedance levels. Am J Gastroenterol 2011;106:2093-2097.

8. Woodland P, Al-Zinaty M, Yazaki E, Sifrim D. In vivo evaluation of acid-induced changes in oesophageal mucosa integrity and sensitivity in non-erosive reflux disease. Gut 2013;62:1256-1261.

9. Martinucci I, De Bortoli N, Savarino E, et al. Esophageal baseline impedance levels in patients with pathophysiological characteristics of functional heartburn. Neurogastroenterol Motil 2014;26:546-555.

10. Frazzoni M, Savarino E, de Bortoli N, et al. Analyses of the post-reflux swallow-induced peristaltic wave index and nocturnal baseline impedance parameters increase the diagnostic yield of impedance-pH monitoring of patients with reflux disease. Clin Gastroenterol Hepatol 2016;14:40-46.

11. Ribolsi M, Savarino E, De Bortoli N, et al. Reflux pattern and role of impedance-pH variables in predicting PPI response in patients with suspected GERD-related chronic cough. Aliment Pharmacol Ther 2014;40:966-973.

12. Rinsma NF, Farré R, Bouvy ND, Masclee AA, Conchillo JM. The effect of endoscopic fundoplication and proton pump inhibitors on baseline impedance and heartburn severity in GERD patients. Neurogastroenterol Motil 2015;27:220-228.

13. de Bortoli N, Martinucci I, Savarino E, et al. Association between baseline impedance values and response proton pump inhibitors in patients with heartburn. Clin Gastroenterol Hepatol 2015;13:1082-1088, e1.

14. Kandulski A, Weigt J, Caro C, Jechorek D, Wex T, Malfertheiner P. Esophageal intraluminal baseline impedance differentiates gastroesophageal reflux disease from functional heartburn. Clin Gastroenterol Hepatol 2015;13:1075-1081.

15. Patel A, Wang D, Sainani N, Sayuk GS, Gyawali CP. Distal mean nocturnal baseline impedance on $\mathrm{pH}$-impedance monitoring predicts reflux burden and symptomatic outcome in gastro-oesophageal reflux disease. Aliment Pharmacol Ther 2016;44:890-898.

16. Frazzoni L, Frazzoni M, de Bortoli N, et al. Postreflux swallow-induced peristaltic wave index and nocturnal baseline impedance can link PPIresponsive heartburn to reflux better than acid exposure time. Neurogastroenterol Motil 2017;29:e13116.

17. Aziz Q, Fass R, Gyawali CP, Miwa H, Pandolfino JE, Zerbib F. Esophageal disorders. Gastroenterology 2016;150:1368-1379.

18. Hanada Y, Hoshino S, Hoshikawa Y, et al. Endoscopic diagnosis of hiatus hernia under deep inspiration is not consistent with esophageal manometric diagnosis. J Gastroenterol 2018;53:712-717.

19. Nakagawa K, Sawada A, Hoshikawa Y, et al. Persistent postprandial regurgitation vs rumination in patients with refractory gastroesophageal reflux disease symptoms: identification of a distinct rumination pattern using ambulatory impedance-pH monitoring. Am J Gastroenterol 2019;114:1248-1255.

20. Shay S, Tutuian R, Sifrim D, et al. Twenty-four hour ambulatory simultaneous impedance and $\mathrm{pH}$ monitoring: a multicenter report of normal values from 60 healthy volunteers. Am J Gastroenterol 2004;99:10371043.

21. Kanda Y. [Statistical analysis using freely-available "EZR (Easy R)" software.] Rinsho Ketsueki 2015;56:2258-2266. [Japanese]

22. Frazzoni M, de Bortoli N, Frazzoni L, et al. Impairment of chemical clearance and mucosal integrity distinguishes hypersensitive esophagus from functional heartburn. J Gastroenterol 2017;52:444-451.

23. Frazzoni L, Frazzoni M, De Bortoli N, et al. Critical appraisal of Rome IV criteria: hypersensitive esophagus does belong to gastroesophageal reflux disease spectrum. Ann Gastroenterol 2018;31:1-7.

24. Rengarajan A, Savarino E, Della Coletta M, Ghisa M, Patel A, Gyawali CP. Mean nocturnal baseline impedance correlates with symptom outcome when acid exposure time is inconclusive on esophageal reflux monitoring. Clin Gastroenterol Hepatol 2020;18:589-595. 